\title{
PERCEPÇÃO DE UMA EQUIPE DE UNIDADE DE TERAPIA INTENSIVA CARDIOLÓGICA ACERCA DA MOBILIZAÇÃO PRECOCE
}

\author{
PERCEPTION OF A CARDIOLOGICAL INTENSIVE CARE UNIT TEAM \\ ABOUT EARLY MOBILIZATION \\ Irllanna Ketley Santos do Nascimento, I* Dyego Anderson Alves de Farias, " Danyelle Nóbrega de Farias, III \\ Matheus dos Santos Soares IV
}

\begin{abstract}
Resumo. As doenças cardíacas estão entre as principais causas de óbito e o tratamento envolve mudanças de hábitos de vida e, em alguns casos, cirurgia. Durante o internamento, complicações podem ocorrer, a exemplo da diminuição da força muscular. A Mobilização Precoce (MP) intervém dando suporte para reverter ou minimizar essas complicações, porém, ainda assim, existem barreiras para sua realização. O objetivo do estudo foi avaliar o conhecimento de uma equipe de Unidade de Terapia Intensiva sobre a MP, evidenciando seus aspectos e possíveis barreiras para realização. Tratou-se de um estudo transversal de caráter observacional com abordagem exploratória, descritiva e delineamento quantitativo realizado com os profissionais da equipe da UTI Cardiológica de um Hospital Universitário na cidade de João Pessoa-PB. Foram entrevistados 27 profissionais das especialidades Fisioterapia, Medicina, Enfermagem e técnico de enfermagem, por meio da aplicação de um questionário estruturado, utilizando-se de respostas em uma escala tipo Likert, assim como de questões abertas. Os resultados evidenciaram que a maioria dos profissionais possui bom entendimento acerca da MP,dos efeitos da imobilidade e da importância do trabalho em equipe. Em adição, a recusa do paciente e a falta de conhecimento sobre riscos e benefícios da realização foram elencadas como barreiras para realização da MP no serviço. Apesar dos resultados, foram identificadas divergências quanto à realização diária da MP, bem como do seguimento de protocolo do serviço, o que evidencia a necessidade de ações educativas continuadas.
\end{abstract}

PALAVRAS-CHAVE: Mobilização Precoce. Unidade de Terapia Intensiva. Unidades de Cuidados Coronarianos.

Abstract. Heart disease is among the main causes of death in Brazil and its treatment can range from lifestyle changes to the need for surgery. Complications may occur during hospitalization, one of which is the decrease in muscle strength. Thus, Early Mobilization (EM) intervenes providing support to reverse or minimize such complications, however, there are still barriers to its realization. Hence, the objective of the study was to assess the knowledge of an Intensive Care Unit team about EM, highlighting its aspects and possible barriers to its execution. This was an observational cross-sectional study with an exploratory, descriptive, and quantitative design carried out with professionals from the Cardiac ICU team of a University Hospital in the city of João Pessoa-PB. 27 professionals from the Physiotherapy, Medicine, Nursing and Nurse Technician areas were interviewed, through the completion of a structured questionnaire using answers on a Likert scale, as well as open questions. Results evidenced that most professionals have a good understanding of Early Mobilization, the effects of immobility and the importance of teamwork, and two barriers were listed for carrying out early mobilization in the service, the patient's refusal, and the lack of knowledge about its risks and benefits. Despite the results, divergences were identified regarding the daily performance of early mobilization and the adherence to the treatment protocol, which highlights the need for ongoing education.

KEYWORDS: Early Mobilization. Intensive Care Unit. Coronary Care Units. 


\section{INTRODUÇÃO}

As doenças cardíacas são caracterizadas como aquelas em que há alteração do sistema cardíaco e vascular, podendo repercutir em outros sistemas que incluem doenças coronarianas, cerebrovasculares, arteriais periféricas, afecções reumáticas e congênitas, além de trombose e embolia. ${ }^{1}$ As doenças cardiovasculares estão entre as maiores causas de óbito no Brasil, com cerca 400 mil casos no ano de 2020 e, além disso, estão relacionadas à maioria dos procedimentos cirúrgicos realizados. ${ }^{2,3}$ Segundo dados do Departamento de Informática do Ministério da Saúde (DATASUS), em 2010, o número de cirurgias cardíacas foi de 103.300, já em 2014, 92.106, sendo 97\% delas realizadas pelo Sistema Único de Saúde. ${ }^{4}$

O tratamento da doença cardíaca é complexo devido ao aspecto multifatorial, envolvendo o uso crônico de medicamentos, prática de atividade física, mudança de hábitos de vida, alimentação adequada, cessação do uso do tabaco e álcool, entre outros. Além disso, pode causar complicações em outros órgãos, como pulmão e rins e que, associadas a outros fatores, comprometem a capacidade funcional do indivíduo. ${ }^{5} \mathrm{~A}$ reabilitação do paciente cardiopata necessita de uma intervenção multidisciplinar, uma vez que as complicações da doença podem ser amplas. Dessa forma, é preciso estabelecer uma forma de se trabalhar o indivíduo como um todo e não visando apenas sua patologia de base. Para tanto, se faz necessário o acompanhamento multiprofissional por médicos, fisioterapeutas, profissionais de educação física, nutricionistas, psicólogos, entre outros.

Esgotados os recursos do tratamento conservador, a depender da gravidade do quadro, a cirurgia cardíaca torna-se um procedimento indicado com o objetivo de melhorar a função ventricular, prevenir infarto e proteger o miocárdio. Apesar dos benefícios, a cirurgia cardíaca pode provocar complicações como instabilidade hemodinâmica, alterações no sistema respiratório e cardiovascular, além da necessidade de cuidados na Unidade de Terapia Intensiva (UTI) que impactam na capacidade funcional do doente. ${ }^{6}$ Entre as consequências mais comuns no pósoperatório de cirurgia cardíaca e, associadas ao tempo de internação na UTI, estão a diminuição dos volumes e capacidades pulmonares e a fraqueza muscular global, em especial nos pacientes em uso da Ventilação Mecânica Invasiva. 7,8

A equipe multiprofissional da UTI, em geral, intervém de forma integrada com suporte intensivo para amenizar ou até abolir condições incômodas adquiridas com a cirurgia e a internação. Assim, a equipe multiprofissional, no pós-operatório, tem o papel de avaliar a condição clínica do paciente, atuar na administração de drogas, avaliar a hemodinâmica, minimizar a ocorrência de complicações respiratórias, orientar e mobilizar precocemente o paciente. ${ }^{9,10,11}$

Diante do contexto, a Mobilização Precoce (MP) destaca-se como uma intervenção imprescindível na reabilitação do paciente internado na UTI, porque contribui na manutenção da força muscular e na funcionalidade do paciente com menores possibilidades de eventos adversos. Neste sentido, o papel do fisioterapeuta na equipe multiprofissional se destaca na condução da MP, em especial pela realização de posicionamentos terapêuticos, de exercícios passivos até a deambulação, treino com carga, 
exercícios respiratórios, entre outros. ${ }^{12,13,14}$

A MP pode contribuir com a diminuição da dispneia e da fadiga muscular. Além do fortalecimento da musculatura respiratória e periférica, contribui com melhora na função cardiorrespiratória, aumenta a distância de deambulação, diminuição do delírium, da inflamação, do tempo da VMI e de internação. $7,15,16,17$ Mas, assim como todo procedimento, existem critérios para execução, além de contraindicações. A execução da MP, em especial no paciente cardiopata, deve ser bem avaliada em virtude da maior probabilidade de instabilidade hemodinâmica desse paciente.

A equipe multiprofissional atuante na UTI deve estar ciente do uso da MP, suas

\section{MATERIAL E MÉTODOS}

Tratou-se de um estudo transversal de caráter observacional com abordagem exploratória, descritiva e delineamento quantitativo realizado, durante o mês de setembro de 2020, na UTI Cardiológica de um Hospital Universitário na cidade de João Pessoa-PB. Participaram da pesquisa profissionais da equipe da UTI formada por enfermeiros, técnicos de enfermagem, médicos e fisioterapeutas, que se incluíam no estudo por serem profissionais que estavam no serviço, aceitaram participar da pesquisa e por formarem a equipe que trabalha com MP. Foram realizadas quatro visitas à unidade hospitalar para coleta de dados dos profissionais da equipe da UTI cardiológica, como forma de ter acesso a toda equipe no rodízio da escala de plantões. Os profissionais foram abordados em seus turnos de serviço e orientados acerca da pesquisa e do TCLE.

Para a realização da coleta de dados, indicações, contraindicações, possíveis eventos adversos e o reconhecimento das reais barreiras encontradas para a intervenção, uma vez que a MP pode minimizar a perda funcional do doente crítico. A MP, por se tratar de um recurso benéfico para a reabilitação de pacientes cardiopatas em ambiente da UTI, como também, no pósoperatório imediato em enfermarias, pode e deve ser utilizada em protocolos de tratamento intensivo, a exemplo do proposto por Marra et al. ${ }^{18}$ que inclui a MP no pacote de recomendações para a libertação do paciente da UTI. Dessa forma, o objetivo do trabalho foi avaliar o conhecimento da equipe da UTI de um Hospital Universitário sobre a $M P$, evidenciando seus aspectos e possíveis barreiras para realização.

foi utilizado um formulário subdividido em itens referentes ao perfil sociodemográfico dos profissionais como sexo e idade, além de informações quanto ao tempo de experiência e capacitações na área da UTI, itens que avaliaram o conhecimento dos profissionais sobre a MP, suas implicações e barreiras do serviço e questões acerca do imobilismo. Esse formulário foi elaborado com base nos trabalhos já publicados, ${ }^{19,16,20,21}$ além do instrumento do Hospital Jhons Hopkins acerca da MP. ${ }^{22}$

Para os itens de avaliação do conhecimento da MP, as respostas corresponderamaumaescaladepossibilidades pré-estabelecidas (tipo Likert), na qual foram atribuídas as respostas: "Concordo totalmente", "Concordo", "Neutro", "Discordo" e "Discordo totalmente". A segunda parte do instrumento consistiu em questões abertas. Para análise dos resultados, 
as respostas "Concordo totalmente" e "Concordo" foram reunidas em um único grupo, da mesma forma que as respostas "Discordo" e "Discordo totalmente", assim como o uso de variáveis discretas para a elaboração da porcentagem.

De forma complementar, um item do instrumento correspondeu a respostas abertas para que os profissionais listassem as principais barreiras e contraindicações do serviço para a realização da MP. A duração da aplicação dos instrumentos variou de acordo com a disponibilidade de tempo do entrevistado.

A pesquisa foi aprovada pelo Comitê de Ética e Pesquisa das Faculdades de Enfermagem e Medicina Nova Esperança, sob n ${ }^{\circ}$ CAAE: 35801220.7 .0000 .5179$.

\section{RESULTADOS E DISCUSSÃO}

Foram entrevistados 27 profissionais, sendo quatro enfermeiros, três fisioterapeutas, 16 técnicos de enfermagem e quatro médicos. Desse total, 22 eram do sexo feminino, com média de idade de 34 anos (Tabela 1). Com relação ao tempo de experiência, $72 \%$ tinham tempo menor do que cinco anos. Já em relação à capacitação, 91\% dos profissionais de nível superior possuíam especialização na área da UTI e $25 \%$ dos profissionais de nível médio relataram ter realizado curso ou capacitação na área.

TABELA 1. Perfil dos profissionais da Unidade de Terapia Intensiva (UTI) Cardiológica de um Hospital Universitário do município de João Pessoa, Paraíba.

\begin{tabular}{lcccccc}
\hline Especialidade & $\begin{array}{c}\text { Número } \\
\text { de } \\
\text { profissionais }\end{array}$ & $\begin{array}{c}\text { Tempo de } \\
\text { Experiência } \\
\text { em UTI <5 anos }\end{array}$ & $\begin{array}{c}\text { Tempo de } \\
\text { Experiência } \\
\text { em UTI>5 anos }\end{array}$ & $\begin{array}{c}\text { Tempo no } \\
\text { serviço } \\
<5 \text { anos }\end{array}$ & $\begin{array}{c}\text { Tempo no } \\
\text { serviço } \\
>5 \text { anos }\end{array}$ & $\begin{array}{c}\text { Capacitação/ } \\
\text { Curso na área } \\
\text { de UTI }\end{array}$ \\
\hline Médicos & 4 & $1(25,0 \%)$ & $3(75,0 \%)$ & $2(50,0 \%)$ & $1(25,0 \%)$ & $4(100,0 \%)$ \\
Enfermeiros & 4 & $4(100,0 \%)$ & $0(0,0 \%)$ & $4(100,0 \%)$ & $0(0,0 \%)$ & $4(100,0 \%)$ \\
Fisioterapeutas & 3 & $1(33,0 \%)$ & $1(33,0 \%)$ & $2(67,0 \%)$ & $1(33,0 \%)$ & $2(67,0 \%)$ \\
Téc. Enfermagem & 16 & $12(86,0 \%)$ & $2(14,0 \%)$ & $9(70,0 \%)$ & $1(33,0 \%)$ & $12(86,0 \%)$ \\
\hline
\end{tabular}

A capacitação e a experiência da equipe no manejo do paciente crítico são fundamentais para um atendimento de qualidade, pois minimiza danos ao paciente durante a internação. $\mathrm{Na}$ pesquisa, foi constatado que a equipe multiprofissional é composta, em sua maioria, por profissionais com tempo de experiência no serviço menor do que cinco anos, mas que realizaram cursos de capacitação e/ou especialização na área. A capacitação se faz importante, pois possibilita atribuir ao profissional mais conhecimento acerca de determinado tema e área, contribuindo, assim, no aprimoramento de suas habilidades e, consequentemente, abrangendo melhor as necessidades durante a assistência. ${ }^{23,24}$

A Tabela 2 apresenta os resultados quanto ao conhecimento dos profissionais sobre a MP. Observou-se uma diversificação de opiniões dos profissionais quanto as consequências do imobilismo e aos princípios da MP. 
TABELA 2. Conhecimento de profissionais da Unidade de Terapia Intensiva (UTI) Cardiológica, sobre Mobilização Precoce (MP) e questões do serviço de um Hospital Universitário do município de João Pessoa, Paraíba.

\begin{tabular}{|c|c|c|c|}
\hline Questões & $\begin{array}{l}\text { Discordo/ } \\
\text { Discordo } \\
\text { Totalmente }\end{array}$ & Neutro & $\begin{array}{l}\text { Concordo/ } \\
\text { Concordo } \\
\text { Totalmente }\end{array}$ \\
\hline Eu conheço a síndrome do imobilismo? & $(n=4) 15,0 \%$ & $4(n=2) 7,0 \%$ & $(n=21) 78,0 \%$ \\
\hline $\begin{array}{l}\text { Entre as complicações do imobilismo estão a diminuição da } \\
\text { força muscular, alterações ósseas, complicações respiratórias e } \\
\text { hemodinâmicas? }\end{array}$ & $(n=1) 4,0 \%$ & $(n=1) 4,0 \%$ & $(n=25) 92,0 \%$ \\
\hline A MP deve ser realizada em todos os pacientes da UTI? & $(n=17) 63,0 \% 3$ & $(n=2) 7,0 \%$ & $(n=8) 30,0 \%$ \\
\hline $\begin{array}{l}\text { A minha equipe da UTI discute sobre a necessidade de MP durante a } \\
\text { rotina do plantão? }\end{array}$ & $(n=7) 26,0 \%$ & $(n=5) 18,0 \%$ & $(n=15) 56,0 \%$ \\
\hline O meu serviço dispõe de protocolo de MP? & $(n=7) 26,0 \%$ & $(n=4) 15,0 \%$ & $(n=16) 59,0 \%$ \\
\hline A MP deve ser avaliada e realizada por toda equipe da UTI? & $(n=1) 4,0 \%$ & $(n=2) 7,0 \%$ & $(n=24) 89,0 \%$ \\
\hline Conheço e compreendo as contraindicações para realização da MP? & $(n=2) 7,0 \%$ & $(n=4) 15,0 \%$ & $(n=21) 78,0 \%$ \\
\hline A MP é realizada diariamente no serviço? & $(n=11) 41,0 \%$ & $(n=4) 15,0 \%$ & $(n=12) 44,0 \%$ \\
\hline
\end{tabular}

Quanto ao conhecimento sobre a MP, a maioria, (92\%) dos profissionais, concorda que a Síndrome do Imobilismo é responsável por uma série de complicações que podem ser refletidas em todos os sistemas orgânicos do paciente e que impactará no desfecho da internação. A Síndrome do Imobilismo ocorre quando o paciente permanece acamado, sem movimento, devido a ventilação mecânica invasiva, sedação e quadro clínico grave, além de outros fatores, o que repercute em perda da força muscular e diminuição de amplitude de movimento. 25

O Imobilismo acarreta danos que podem perdurar por semanas ou chegar a meses e anos. Para minimização destes agravos, a ação da equipe da UTI na realização da MP se faz necessária, pois é importante que os profissionais tenham conhecimento sobre o tema para que trabalhem de forma preventiva e correta. 25,26

Os protocolos de libertação do paciente da UTI recomendam que os profissionais discutam diariamente a MP no ambiente hospitalar, sendo atribuição de toda equipe, uma vez que a MP tem papel crucial no processo de recuperação do doente crítico. $^{27,28}$ Do total de entrevistas, $56 \%$ referiram que a equipe discute diariamente anecessidade de MP e 59\% concordaram que o serviço dispõe de protocolo de MP (Tabela 2).

Diante do exposto, enfatiza-se a necessidade de que o protocolo seja priorizado na assistência, além de ser discutido como forma de reciclar o conhecimento da equipe sobre o tema. Além disso, $44 \%$ concordam que a MP é realizada diariamente no serviço e $41 \%$ discordam, além dos $14 \%$ neutros. A contrariedade das respostas pode evidenciar que o protocolo não é de conhecimento de todos. Os protocolos nos serviços são importantes, pois facilitam, uniformizam e garantem a continuidade da assistência, auxiliando o desempenho do serviço, sendo também um estímulo ao paciente, evidenciando que, mesmo com as barreiras, é importante que sejam seguidos, com maior frequência, implementações de programas como esse. ${ }^{29,30}$

Quando os profissionais foram questionados se a MP deve ser realizada em 
todos os pacientes, ocorreu uma divergência entre as repostas. Cerca de $63 \%$ discordaram da indicação ampla da MP e dois profissionais responderam de forma neutra. Este achado não corrobora o mencionado sobre os efeitos deletérios do Imobilismo.

A MP é uma intervenção relevante no ambiente de UTI e faz parte do Bundle $A B C D E F$ que auxilia numa intervenção focal e efetiva da equipe, o que possibilita o melhor manejo do doente crítico, diminuição de custos à saúde e alta precoce, por isso, a importância de seu uso nesse ambiente. O pacote enfatiza a importância da MP no processo de recuperação do paciente e ela associada às demais intervenções da equipe contribuem significativamente com a condição de saúde do paciente e minimizam a perda funcional, por meio do movimento. ${ }^{31} \mathrm{~A}$ MP pode ser realizada a partir de um simples posicionamento terapêutico até a realização da deambulação do paciente. Dessa forma, independentemente do quadro do paciente, condutas poderão ser realizadas.

A Tabela 3 reporta as barreiras do serviço para a realização da MP. Dos 27 entrevistados, dois não responderam todos os itens. Logo, os resultados apresentados representam 25 profissionais. As principais barreiras elencadas foram a recusa do paciente e a falta de conhecimento sobre os riscos e benefícios da MP. As outras barreiras não foram elencadas devido a porcentagem de opções "discordo/discordo totalmente", sendo elas, o tempo reduzido da equipe para a realização da MP (42\%), a mobilização vista como atribuição do fisioterapeuta (70\%), o receio/medo da equipe em mobilizar (60\%), pacientes sem perfil para MP (56\%) e não ser prioridade (63\%).

TABELA 3. Percepção acerca das barreiras do serviço para realização da Mobilização Precoce (MP) na da Unidade de Terapia Intensiva (UTI) Cardiológica de um Hospital Universitário do município de João Pessoa, Paraíba.

\begin{tabular}{lccc}
\hline O serviço possui algumas barreiras para realização da MP, como: & $\begin{array}{c}\text { Concordo/ } \\
\text { Concordo } \\
\text { Totalmente }\end{array}$ & Neutro & $\begin{array}{c}\text { Discordo/ } \\
\text { Discordo } \\
\text { Totalmente }\end{array}$ \\
\hline Recusa do paciente & $13(50,0 \%)$ & $5(19,0 \%)$ & $8(31,0 \%)$ \\
Tempo reduzido da equipe para realização da MP & $6(25,0 \%)$ & $8(33,0 \%)$ & $10(42,0 \%)$ \\
A MP é papel do Fisioterapeuta & $8(30,0 \%)$ & $0(0,0 \%)$ & $19(70,0 \%)$ \\
Receio/ medo da equipe em mobilizar os pacientes & $9(33,0 \%)$ & $2(7,0 \%)$ & $2(7,0 \%)$ \\
Falta de conhecimento sobre riscos e benefícios da MP & $13(48,0 \%)$ & $6(22,0 \%)$ & $8(30,0 \%)$ \\
Pacientes da UTI sem perfil para MP & $7(26,0 \%)$ & $5(18,0 \%)$ & $15(56,0 \%)$ \\
A MP não é prioridade & $6(22,0 \%)$ & $4(15,0 \%)$ & $17(63,0 \%)$ \\
\hline
\end{tabular}

As barreiras para a MP podem ser divididas em barreiras do serviço e dos profissionais, destacando-se a falta de priorização do serviço, responsabilidade apenas do fisioterapeuta, falta de recursos, suposições não avaliadas do que pode acontecer, desconhecimento sobre o tema, falta de esforço multidisciplinar e comunicação, a não valorização da prática, recusa do paciente, suposições acerca da necessidade de repouso, ordens ortopédicas, necessidade de mais profissionais e falta de tempo. $7,15,20,21 \mathrm{Na}$ pesquisa, as principais barreiras referidas pelos profissionais foram a recusa do paciente e falta de conhecimento sobre riscos e benefícios da MP. De fato, existe a necessidade de se respeitar a opção do paciente, no entanto, a equipe deve buscar 
ativamente a possibilidade de realização, haja vista os benefícios.

O entendimento sobre as indicações e contraindicações da MP é fundamental para a minimização da ocorrência de eventos adversos. Entre as contraindicações da MP, destacam-se: tromboflebite, infecção sistêmica aguda, embolia recente, instabilidade hemodinâmica, o que torna fundamental o trabalho interdisciplinar para sua resolução. ${ }^{11} \mathrm{Em}$ relação a pesquisa, as principais contraindicações citadas foram a instabilidade hemodinâmica, pacientes graves, recusa do paciente e pós-operatório imediato de angioplastia.

Devido aos profissionais trabalharem numa UTI cardiológica e sabendo-se que no pós-operatório imediato de angioplastia há a necessidade de manutenção do paciente em decúbito zero, com repouso nas primeiras seis horas pelos riscos de sangramento, equimose e fístula arteriovenosa, pode-se presumir o

\section{CONCLUSÃO}

A Mobilização Precoce (MP), enquanto parte do pacote de libertação da UTI, evidencia sua importância e os benefícios que podem levar ao paciente e ao serviço. Deste modo, é necessário que os profissionais da equipe da UTI compreendam, discutam sobre o tema e trabalhem de forma coletiva e integrada, pois a realização dela é de responsabilidade de todos os profissionais que compõem a equipe da UTI, seja na execução ou, na facilitação da sua realização. Dessa forma, são importantes a elaboração e o seguimento de um protocolo de MP do serviço, pois eles podem facilitar a rotina do serviço assim como no manejo do paciente.

O presente estudo abordou além motivo de a maioria dos profissionais citarem a angioplastia como contraindicação. ${ }^{32,33} \mathrm{~A}$ divergência entre a realização diária da MP também pode ser explicada, em parte, pelo mesmo motivo, além do que, a depender do número de admissões de pacientes e da realização de procedimentos cirúrgicos, a UTI pode atender a um público maior de pacientes com o perfil da angioplastia.

A MP pode ser conduzida por meio de exercícios passivos no próprio leito, exercícios ativos, deambulação, exercícios com carga, treinamento muscular respiratório, ${ }^{13,34}$ o que corrobora as principais condutas referidas pelos entrevistados, no qual destacam-se a sedestação no leito, mudanças de decúbito e deambulação. Alguns profissionais associaram a MP com a necessidade de contenção do paciente no leito, o que reflete, ainda mais, a necessidade de capacitação dos profissionais quanto ao tema.

da MP, também o Imobilismo, aspectos contribuintes para manejo da equipe na UTI, contribuições desses profissionais na intervenção direta ao paciente do setor e pôde evidenciar a compreensão deles a respeito de alguns temas.

A maioria dos profissionais da equipe compreendem as complicações do Imobilismo e a importância da MP. No entanto, alguns profissionais não souberam discutir acerca de questões relevantes, além da divergência da equipe quanto à realização diária da mobilização e do seguimento do protocolo do serviço, o que evidencia uma necessidade de educação continuada. 


\section{REFERÊNCIAS BIBLIOGRÁFICAS}

1. ORGANIZAÇÃO PAN AMERICANA DA SAÚDE - OPAS. Doenças Cardiovasculares.2017 disponível em: https://www.paho.org/bra/index. php?option=com_content\&view=article\&id=5253:d oencas-cardiovasculares\&ltemid=1096.

2. Garzesi AM, Garcia LR, Felicio ML, Santos AAT, Passaroni AC, Martins AS, et al. Capítulo 4,Ebook Cirurgia Cardíaca do Adulto. Departamento de Cirurgia e Ortopedia da Faculdade de Medicina de Botucatu - UNESP. São Paulo, 2018.

\section{SOCIEDADE BRASILEIRA DE CARDIOLOGIA.} Cardiomêtro. 2020. Disponível em: http://www. cardiometro.com.br/grafico.asp; acesso realizado em 15/06/2021.

4. BRASIL. Departamento de Informática do Sistema Único de Saúde. Cuidado às Pessoas com Doenças Cardiovasculares no SUS, 2015.

5. Soares GMT, Ferreira DCS, Gonçalves MPC, Alves TGS, David FL, Henriques KMC, et al. Prevalência das Principais Complicações Pós-Operatórias em Cirurgias Cardíacas. Rio de Janeiro:Rev Bras Cardiol. 2011 Mai/jun; 4(3):139-46.

6. Oliveros MJ, Sepúlveda $P$, Serón P, Fuentes R.Rehabilitación Cardíaca Fase I: Progresión segúnpasos protocolizados en pacientes cardioquirúrgicos em un hospital público de Chile. Chile: RevistaChilena de Cardiología. 2019Abr; 38(1): 9-19.

7. Harrold ME, Salisbury LG, Webb SA, Alisson GT. Early mobilisation in intensive care units in Australia and Scotland: a prospective, observational cohort study examining mobilisation practices and barriers. ReinoUnido:Crit Care.2015 Sep; 19(1):336.

8. Parry SM, Huang M, Needham DM. Evaluating physical functioning in critical care: considerations for clinical practice and research.ReinoUnido: Crit Care. 2017 Oct; 21(1):249.

9. Amorim TV, Salimena AMO. Processo cirúrgico cardíaco e suas implicações no cuidado de enfermagem: revisão/reflexão. Juiz de Fora/ Minas Gerais: HU Revista. 2015Jul/dez; 4(3):149-54.

10. García RJV, Escalona ÁLO, Ortiz OMG, García IDV. Necesidades de aprendizaje de médicos de atención primaria sobre reperfusión coronaria em el infarto agudo delmiocardio. Cuba: MEDISAN. 2019 Mar/abr; 23(2):232-45.

11. EBSERH. Fisioterapia no Pós-Operatório de Cirurgia Cardíaca no Paciente Adulto. Protocolo Operacional Padrão, 2018.

12. Conceição TMA, Gonzáles AI, Figueiredo FCXS, Vieira DSR, Bündchen DC. Critérios de segurança para iniciar a mobilização precoce em unidades de terapia intensiva. Revisão sistemática. São Paulo: Rev Bras Ter Intensiva. 2017 Dez; 29(4): 509-19.

13. Santos GO, Queiroz RS, Jesus CS, Carneiro JAO, Faria LMA, Fernandes MH, et al. Pacientes internados em unidade de terapia intensiva que não adotam postura antigravitacional apresentam maiores chances de óbito. São Paulo:Fisioter. Pesqui. 2019 Set; 26(2): 235-40.

14. Silva VS, Pinto JG, Martinez BP, Camelier FWR. Mobilização na Unidade de Terapia Intensiva: revisão sistemática. São Paulo: Fisioter. Pesqui. 2014 Out/ dez; 24(4):398-04.

15. Truong AD,Fan E, Brower RG, Needham DM.Bench-to-bedside review: Mobilizing patients in the intensive care unit - from pathophysiology to clinical trials. ReinoUnido: Crit Care. 2009 Jul; 13(4).

16. Dubb R, Nydahl P, Hermes C, Schwabbauer N, Toonstra A, Parker AM, et al. Barriers and Strategies for Early Mobilization of Patients in Intensive Care Units.EstadosUnidos: Ann Am Thorac Soc. 2016 May;15(5): 724-30.

17. Hodgson CL, Capell E, Tipping, CJ. Early Mobilization of Patients in Intensive Care: 
Organization, Communication and Safety Factors that Influence Translation into Clinical Practice. ReinoUnido: Crit Care. 2018 Mar; 77(22).

18. Marra A, Ely EW, Pandharipande PP, Patel MB.The $A B C D E F$ Bundle in Critical Care. Estados Unidos: Critical Care Clinics. 2017 Apr;33(2): 225-43.

19. Fontela PC, Forgiarini JR LA, Friedman G. Atitudes clínicas e barreiras percebidas para a mobilização precoce de pacientes graves em unidades de terapia intensiva adulto. São Paulo: Rev Bras Ter Intensiva. 2018 Jun; 30(2): 187-94.

20. Hoyer EH, Brotman DJ, Chan KS, Needham DM. Barriers to Early Mobility of Hospitalized General Medicine Patients. Estados Unidos: Am J Phys Med Rehabil. 2015 Apr; 94(4): 304-12.

21. Goodson CM, Friedman LA, Mantheiy E, Heckle K, Lavezza A, Toonstra A, et al. Perceived Barriers to Mobility in a Medical ICU: The Patient Mobilization Attitudes \& Beliefs Survey for the ICU. Cambridge: J Intensive Care Med. 2018.

22. The Johns Hopkins Medicine. Patient Mobilization Attitudes \& Beliefs Survey.Activity and Mobility Promotion.20--?Site para busca: https:// www.hopkinsmedicine.org/physical_medicine rehabilitation/education_training/amp/toolkit.html

23. Dias DM, Guimarães MGV. Avaliação do impacto da capacitação profissional no ambito da prefeitura de Manaus por meio da aplicação do modelo impact. João Pessoa: Perspectivas em Gestão \& Conhecimento. 2016 Jan/Jun; 6(1): 200-22.

24. Martins FR, Morini MS, Olinda AG, Barros FHV, Silva LO, Roseno MASG. Necessidades de qualificação do processo de trabalho da Enfermagem em UTI Pediátrica. Brasil: Rev. Mult. Psic. 2019; 13(43): 322-28.

25. Pereira HCB, Duarte PHM, Mélo TM, Silva RMC, Santos WV, Barbosa DS, et al.Intervenção fisioterapêutica na Síndrome da Imobilidade empessoas idosas: revisão sistematizada. São Paulo: Archives of Health Investigation. 2017; 6(11): 505-08.
26. Castelino T, Fiore JF, Niculiseanu P, Landry T, Augustin B, Feldman LS. The effect of early mobilization protocols on postoperative outcomes following abdominal and thoracic surgery: A systematic review. Holanda: Surgery - Elsevier. 2016 Apr; 159(4): 991-03.

27. Hodgson CL, Stiller K, Needham DM, Tipping CJ, Harrold M, Baldwin CE, et al. Expert consensus and recommendations on safety criteria for active mobilization of mechanically ventilated critically ill adults. ReinoUnido: Crit Care. 2014 Dec; 18(6): 658.

28. Stollings JL, Devlin JW, Lin JC, Pun BT, Byrum D, Barr J. Best practices for conducting interprofessional team rounds to facilitate performance of the ICU liberation $(A B C D E F)$ bundle. Nova York: Crit. Care Med. 2020 Apr; 48(4): 562-70.

29. Rupich K, Missimer E, OBrien D, Shafer G, Wilensky EM, Pierce JT, et al. The Benefits of Implementing an Early Mobility Protocol in Postoperative Neurosurgical Spine Patients. Nova York: Am J Nurs. 2018 Jun; 118(6): 46-53.

30. Dirkes SM, Kozlowski C. Early Mobility in the Intensive Care Unit: Evidence, Barriers, and Future Directions. Secaucus: Crit Care Nurse. 2019 Jun; 39(3): 33-43

31. ICU Liberation.The ABCDEF Bundle. Disponível em: http://www.iculiberation.org . Acesso dia 10 de Outubro de 2020.

32. Aguiar GA, Aguiar MA. Angioplastia Coronária: desafios e possibilidades na assistência de Enfermagem. Brasil: DESAFIOS-Revista Interdisciplinar Da Universidade Federal Do Tocantins. 2019; 6(1): 87-89.

33. Lima VCGS, Queluci GC, Brandão ES. Cuidados de enfermagemao cliente pós-angioplastia transluminal coronariana. Recife: Rev. enferm. UFPE on line. 2019 Mar; 13(3): 732-42.

34. Paton M, Lane R, Hodgson CL.Early Mobilization in the Intensive Care Unit to Improve Long-Term Recovery.EstadosUnidos: Crit Care Clin.2018 Aug/ oct; 34(4): 557-71. 